\title{
Fabrication of nanostructured transmissive optical devices on ITO-glass with UV1116 photoresist using high-energy electron beam lithography
}

\author{
Calum Williams, Richard Bartholomew, Girish Rughoobur, \\ George Gordon, Andrew Flewitt, Timothy Wilkinson \\ Electrical Engineering Division, Department of Engineering, University of \\ Cambridge, 9 JJ Thomson Avenue, Cambridge CB3 0FA, United Kingdom \\ E-mail: cw507@cam.ac.uk
}

\begin{abstract}
.
High-energy electron beam lithography for patterning nanostructures on insulating substrates can be challenging. For high resolution, conventional resists require large exposure doses and for reasonable throughput, using typical beam currents leads to charge dissipation problems. Here, we use UV1116 photoresist [Dow Chemical Company], designed for photolithographic technologies, with a relatively low area dose at a standard operating current $\left(80 \mathrm{kV}, 40-50 \mu \mathrm{C} \mathrm{cm}^{-2}, 1 \mathrm{nAs}^{-1}\right)$ to pattern over large areas on commercially coated ITO-glass cover slips. The minimum linewidth fabricated was $\sim 33 \mathrm{~nm}$ with $80 \mathrm{~nm}$ spacing; for isolated structures, $\sim 45 \mathrm{~nm}$ structural width with $50 \mathrm{~nm}$ separation. Due to the low beam dose, and $\mathrm{nA}$ current, throughput is high. This work highlights the use of UV1116 photoresist as an alternative to conventional e-beam resists on insulating substrates. To evaluate suitability, we fabricate a range of transmissive optical devices, that could find application for customized wire-grid polarisers and spectral filters for imaging, which operate based on the excitation of surface plasmon polaritons in nanosized geometries, with arrays encompassing areas $\sim 0.25 \mathrm{~cm}^{2}$.
\end{abstract}




\section{Introduction}

Nanostructured transmissive optical devices have significant potential in a number of fields such as spectral filters for imaging, high extinction ratio wire-grid polarisers and high-efficiency diffractive optical elements etc. In addition, plasmonic-enhanced devices, whereby dimensions are highly sub-wavelength, have recently gained a wealth of interest, offering unique functionality in novel optical devices including plasmonic color filters, metasurfaces, holographic elements etc. [1-5]. Surface plasmons (SPs) are collective free-electron oscillations at metal-dielectric interfaces, excited through the applied electric field of incident radiation. This excitation leads to a large electric field enhancement and can be achieved in one, two or three dimensions depending on the geometry and material parameters [6]. In order to fabricate elements with features $<<\lambda$, electron beam lithography (EBL) is commonly used due to its superior resolution capability $(<10 \mathrm{~nm})$, in comparison to UV-based photolithography. However, EBL is low throughput and for patterning on non-conducting transparent substrates, write times increase as to alleviate the associated charge dissipation problems, beam current is reduced. Hence, throughput decreases and total exposure areas are only ever small. In contrast, photolithography is fast, yet cannot achieve the resolution capability of EBL (diffraction limited). In this paper we utilize UV1116 (0.5) photoresist [Dow Chemical Company] (due to its low critical dose and good processing windows), in combination with high-energy EBL, to demonstrate the patterning of dense, complex geometry, subwavelength metallic features on indium-tin-oxide (ITO)-glass substrates over areas 0.25 $\mathrm{cm}^{2}$ with short write times. This work offers an attractive route toward large-area fabrication of nanostructured transmissive optical elements using high-energy EBL.

High-energy (EBL) offers a range of advantages over low-energy systems, including proximity effect reduction, vertical resist sidewalls and dimension accuracy over rough surfaces $[7,8]$. Nanofabrication on insulating substrates using high energy EBL can be challenging due to several key factors. These include increased electron back scattering; insufficient charge dissipation; large area dose required for exposing conventional high resolution e-beam resists (such as poly(methyl methacrylate) (PMMA) / hydrogen silsesquioxane (HSQ) / ZEP-series) at high EBL acceleration voltages; and inevitably a large beam current in order to pattern with acceptable throughput [7-16]. Workarounds include depositing an ultra-thin metallic layer either above or below the resist or using a conductive polymer; both methods are used to dissipate the built-up charge during exposure [10,17-20]. A low beam current can also be utilized in order to suppress the charging effect, however this in itself results in low throughput and subsequently limiting it's applicability to manufacturing of any significant scale. In addition, because resist dose windows scale with beam energy, the required exposure dose for PMMA / HSQ / ZEP at high acceleration voltages $(\sim 100 \mathrm{kV})$ is such that large-area, dense, high resolution feature designs result in long write-times (low throughput) [7,10,12,21]. Alternatively, attempts have been made to use low energy EBL for insulating substrates [17] or sparse, extremely small area patterns [22,23]; yet low energy EBL presents its 
own series of problems including an increase in beam size, the necessity to use very thin resists, pronounced proximity effect etc. [7-9].

UV-grade photoresist is generally implemented on photolithographic systems with relatively large resolution features and hence use in high-resolution applications with EBL is somewhat limited. Nevertheless, because the resist sensitivities are relatively high compared to e-beam resists, hence low area doses are required (leading to short write-times), UV photoresists are of interest for EBL processing, in particular on insulating substrates. SU-8 is a common, extensively used, chemically amplified epoxy based resist. Its use with $100 \mathrm{kV}$ EBL for high resolution features has been investigated elsewhere through the patterning of $\sim 30 \mathrm{~nm}$ lines with $300 \mathrm{~nm}$ pitch on conducting silicon substrates, with an optimized area dose of $20 \mu \mathrm{C} \mathrm{cm}^{-2}$ (thinnest formulation used) [22, 23]. However, there is no literature on the patterning of more complex, sub-wavelength features, on insulating substrates. UV1116 photoresist [Dow Chemical Company] is a high temperature, positive-tone, DUV (KrF-248nm) chemically amplified photoresist. It has good process windows has been shown to offer resolution down to $\sim 30 \mathrm{~nm}$ with a contrast ratio $\sim 10$ on [24] on conductive substrates. Therefore, it has potential as an alternative resist of choice for EBL processing on insulating substrates.

In this work, we explore the potential of UV1116 (0.5) photoresist (low dose) as an alternative to conventional resists for large area, high resolution, high throughput, high energy EBL patterning on insulating substrates. We achieve this through the fabrication of a range of nanostructures on commercial ITO-coated glass without registration marks, using low-dose exposure conditions and nA current. To demonstrate applicability, we fabricate a range of optical nanostructures over large areas, designed to display unique optical phenomena only at certain size scales i.e. localized surface plasmon resonance (LSPR) and SPR-based structures.

\section{Considerations for EBL on insulating substrates}

In contrast to conducting substrates, when exposing insulating substrates with an electron beam, the charge can become trapped near the surface, is unable to dissipate, resulting in charge build up and subsequently deflecting additional incoming electrons. This results in distorted patterns and loss in resolution $[11,21,25]$. A conductive layer, either above / below the resist, is commonly used in conjunction with patterning on insulating substrates - illustrated in Figure 1. This takes the form of an ultrathin $(<10 \mathrm{~nm})$ layer of metal such as aluminum, which can be removed prior to resist development or in some instances the developer solution is used as a wet etchant. Alternatively, a conductive polymer (such as PEDOT:PSS) or conductive resist can be used [11]. It is important to note that a conductive layer will reduce the resist resolution due to additional electron scattering events (more pronounced with a top-layer) [21] .

ITO has electrical conductivity $\sim 10^{4} \Omega \mathrm{cm}$ and can act as the charge dissipation layer underneath the photoresist. Optically, ITO exhibits $>80 \%$ transmittance in the visible part of the spectrum, however with a thickness $\leq 50 \mathrm{~nm}$, there is a limit to how 
fast the deposited charge can be dissipated. In addition, because ITO has the benefit of being optically transparent, the requirement to remove the conductive layer during / post development is less critical. A low beam current increases the efficiency for charge dissipation, and the associated spot size is smaller at $5 \mathrm{~nm}$ (improved resolution). However for a given exposure area dose the write time will inevitably increase, hence there is a trade-off.

Higher acceleration voltage $(\sim 100 \mathrm{kV})$ EBL offers higher resolution capability owing to the associated 'pencil sharp' beam profile through the resist. This is due to the reduction of forward-scattering events as the electron beam enters the resist, which ensures highly anisotropic resist sidewalls post-development [7, 26, 27]. However, in comparison to low voltage EBL, back-scattering events in the substrate and resist increase, which leads to the proximity effect being more pronounced and increases the probability of unwanted exposure of the resist. When the electron energy in the beam increases, the associated inelastic collision cross-section decreases proportionally. Hence, for positive-tone resists, this decreases the number of polymer-chain scissions and means the required exposure dose increases proportionally to voltage (sensitivity decrease) $[9,27,28]$. Subsequently, the dose window increases however there is a lower overall throughput. Furthermore, when using a thin metallic layer to dissipate charge for insulating substrates, the presence of the metal layer increases the resist clearing dose [18].

For high resolution patterning with a high energy beam, over large insulating areas $\sim 0.1-1 \mathrm{~cm}^{2}$ (with an approximate pattern exposure fill factor $\sim 10-50 \%$ ) in a reasonable timeframe, a photoresist is required with: high sensitivity, in order to use a low beam current (for reduced charging) and thus low area dose for total write time purposes; high intrinsic resolution capability; and standard processing windows. UV1116 therefore offers the potential as an alternative resist choice for EBL processing on insulating substrates and is investigated in this work. A comparison of the typical dose windows for UV1116 and conventional EBL resists (taken from [26]) are shown in Figure 1 (c).

\section{Experimental details}

\subsection{Fabrication recipe and characterisation}

Commercial ITO-coated $(\sim 40-50 \mathrm{~nm})$ cover glass slides (thickness $725 \mu \mathrm{m})$ are manually cut to $\sim 1 \mathrm{~cm}^{2}$ pieces. Under the optical microscope, the ITO layer is nonoptimal, i.e. the ITO layer includes scratches, debris and is not entirely uniform over

the surface. No registration marks are used as part of the processing, a focal plane represented by a polynomial based on 4 separate focus values at the corners of the sample is used. UV1116 (0.5) photoresist [Dow Chemical Company] in conjunction with a Nanobeam nB5 EBL system (NanoBeam ltd.), are used for all exposures. With regards to proximity correction, test patterns are dose corrected but not shape modified. At 


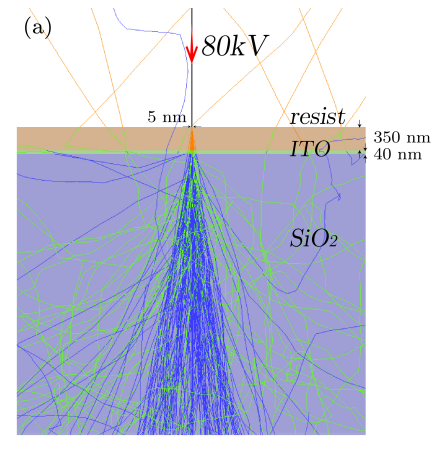

(b)

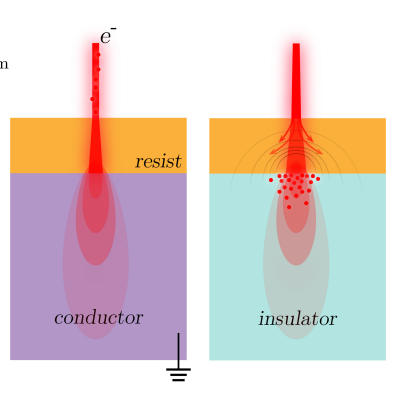

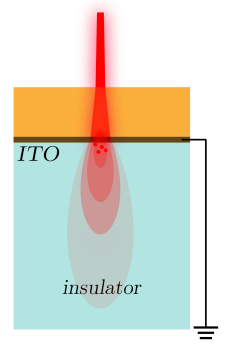

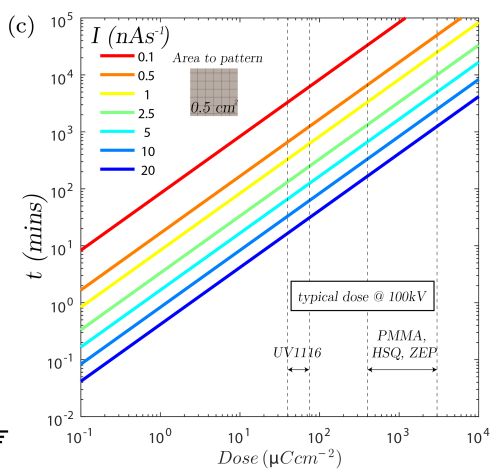

Figure 1. (a) Monte-Carlo (CASINO) [29] simulation of $50080 \mathrm{kV}$ electron trajectories (5 nm spot size ) incident on a polymer resist-ITO-glass structure. Trajectories colored by region (resist / ITO / glass). (b) Charging effect schematically illustrated on insulating substrates. (c) Estimated write-time comparison of different resists and UV1116 with varying exposure currents

$\sim 350 \mathrm{~nm}$, the baked resist thickness is relatively large compared to ultra-high resolution resists such as PMMA ( 100 nm), which will fundamentally limit resolution. MF-CD26 (full concentration) is used as the developer solution. Over multiple samples, the area dose is swept between $40-100 \mu \mathrm{C} \mathrm{cm}^{-2}$, developing times ranged from $12-60 \mathrm{~s}$. Apertures (main and sub-field) and beam currents were kept constant. The fabrication recipe found to offer the highest resolution, but with good reproducibility, is detailed in Table 1.

The optical characterization results are obtained using a BX-51 Olympus polarising optical microscope (Halogen light source with IR filters removed), 10-100x objectives, attached via $200 \mu \mathrm{m}$ optical fiber to a spectrometer (Ocean Optics HR2000+). The spectra are normalized to transmission through an equally thick ITO-glass slide. A Carl-Zeiss scanning electron microscope (SEM) at $1-5 \mathrm{keV}$ is used for imaging the samples.

\subsection{Overview of resolution capability}

Figure 2 shows a collection of the highest resolution metal structures (22nm silver thickness) achieved on ITO-glass using UV1116(0.5) resist: (a) shows a series of photos of a range of samples showing examples of the total areas patterned and the appearance by eye, with (b) and (c) showing a range of isolated features and line tests. At $80 \mathrm{kV}$ exposure, to test minimum linewidths (critical dimension (CD)), a series of logarithmically reducing spaced lines for varying linewidths and duty cycles were patterned - Figure 3 . The minimum achievable linewidth was $\sim 33 \mathrm{~nm}$ with $80 \mathrm{~nm}$ spacing, slightly larger than in [24] but on different substrates. The lowest dose gave the best isolated examples of resolution, and optimal spacing. However smaller linewidths, with larger spacings were hard to achieve with the lower dose. The higher the dose, the more reproducible the line arrays, however this came at the cost of resolution (CD 
Table 1. Fabrication recipe with UV1116 (0.5)

\begin{tabular}{|c|c|}
\hline Process & Conditions \\
\hline Sample cleaning & Ultrasonic baths: acetone, IPA (20mins), $\mathrm{N}_{2}$ blow dry \\
\hline Dehydration bake & $360^{\circ} \mathrm{C}$ for $20 \mathrm{mins}$ (ambient), allow to cool \\
\hline Ensure grounding & $\mathrm{Ag}$ epoxy applied to edges. Bake at $200^{\circ} \mathrm{C}$ (10mins). Ensures electrical grounding \\
\hline Spin-coating & $5,500 \mathrm{rpm}$ for $45 \mathrm{~s}$. Bake at $130^{\circ} \mathrm{C}(2 \mathrm{mins}), \sim 350 \mathrm{~nm}$ resist thickness. \\
\hline EBL & $\begin{array}{l}\text { Exposure: } 80 \mathrm{kV} \text {, area dose } 45 \mu \mathrm{C} \mathrm{cm}^{-2} \text {. Current } 1 \mathrm{nAs}^{-1} \\
\text { Apertures: } 100 \mu \mathrm{m} \text { main-field, } 10 \mu \mathrm{m} \text { sub-field } \\
\text { Deflection: } 40-50 \mathrm{MHz}\end{array}$ \\
\hline Post-exposure bake & $\begin{array}{l}130{ }^{\circ} \mathrm{C} \text { for } 2 \mathrm{mins} \\
\mathrm{MF}-\mathrm{CD}-26 \text { developer (room temp, no dilution) for } 12 \mathrm{~s} \\
\text { DI-water rinse (descum), } \mathrm{N}_{2} \text { blow dry }\end{array}$ \\
\hline Thermal evaporation & Silver $\left(22 \mathrm{~nm}-\right.$ no adhesion layer), Base pressure $1 \times 10^{-6} \mathrm{mbar}$, rate $0.1 \mathrm{nms}^{-1}$ \\
\hline Resist Lift-off & $\begin{array}{l}\text { N-Methyl-2-pyrrolidone (NMP) soak, } 60{ }^{\circ} \mathrm{C} \text { for } 4 \text { hours } \\
\text { NMP sonication for } 1 \text { min, acetone, IPA, } \mathrm{N}_{2} \text { blow dry }\end{array}$ \\
\hline
\end{tabular}

and spacing). For isolated structures, Figure 2 and Figure 4, the smallest features obtainable were $\sim 45 \mathrm{~nm}$ structural width and $50 \mathrm{~nm}$ separation. An area dose of 40 $50 \mu \mathrm{C} \mathrm{cm}{ }^{-2}$ and 10-15s developing time was found to be the optimum for the highest resolution. The development time upper value was $60 \mathrm{~s}$, at this time, the majority of the patterns were not apparent, only some of the larger features. We found $<20$ s gave satisfactory results, however 10-15s gave the smallest features. A dose of $\sim 50-60$ $\mu \mathrm{C} \mathrm{cm}{ }^{-2}$ offered more reproducible results over larger areas but meant a reduction in resolution. The sparsely spaced lines were more reproducible at higher beam energies, but broadened. Approaching $100 \mu \mathrm{C} \mathrm{cm}^{-2}$, isolated features, separated by a minimum $\sim 100 \mathrm{~nm}$, were achievable, however the denser features were not. Figure 4 shows a two sets of patterns, a USAF resolution test target, with shrinking feature sizes (a), and an array of anisotropic nanostructures (b), with varying area dose exposures (12s developing time). For the resolution target, the minimum rulings are nearly achievable with lowest dose but become highly rounded as dose increases. For the nanorod arrays, the features are reasonable at the highest dose, yet the lateral dimensions have expanded, specifically the width, which has broadened more so than its length.

Generally, on ITO-glass we found the critical dose approximately an order of magnitude less than high resolution resists as PMMA and HSQ, and less than ZEP [26]. However, with UV1116, the CD is larger. It is important to note that the work here is on a non-polished insulating substrate, without registration marks and without the lowest available viscosity resist (thinnest resist generally yields higher resolution). Hence, with further optimization, the CD associated with UV1116 should be reduced. Furthermore, with regards to throughput, we experimented with a current of $1 \mathrm{nAs}^{-1}$ and $5 \mathrm{nAs}^{-1}$, (ideally, the largest tolerable beam current should be used) the latter lead to poor resolution results features but was acceptable with larger features $>500 \mathrm{~nm}$. Due to the high sensitivity of UV1116, reasonable throughput was achieved with $1 \mathrm{nAs}^{-1}$, however 
further optimization is possible through sweeping the beam current (optimization) in order to reduce write times. Nevertheless, in context, even with these conditions, in reference to Figure 1 - with comparisons to PMMA and HSQ, with a constant beam current, the write time is approximately an order of magnitude less with UV1116 (if the required $C D$ is $>30 \mathrm{~nm}$ ). With further optimization, this should be improved.

\section{Optical nanostructures}

\subsection{Ultrathin $1 D$ plasmonic gratings}

To evaluate the resolution capability, suitability and throughput of UV1116-based processing, we fabricated a range of 1D ultra-thin plasmonic gratings with a range of linewidths (75 - $220 \mathrm{~nm}$, designed) and duty cycles (0.2 - 0.6, designed), over a range of exposure doses $\left(45-100 \mu \mathrm{C} \mathrm{cm}{ }^{-2}\right)$ and development times (12 - 60s). Commonly these types of grating are fabricated using focused ion beam (FIB) techniques [30,31] due to the discussed problems of large area EBL patterning on insulating substrates.

The experimental results of a range of $\mathrm{Ag}$ (22nm thick) gratings, for 3 different doses are shown in Figure 5; development time is 12s (due to superiority under inspection). SEM images of a range of $1 \mathrm{D}$ gratings at $45 \mu \mathrm{C} \mathrm{cm}^{-2}$ are shown in (b), in relation to their optical counterparts in (c) and transmission spectra in (d). The 1D gratings act as polarisation dependent transmission subtractive color filters. In Figure 5, d (i-vi), for each duty cycle, the linewidth is iterated, (smaller-to-larger), the resulting spectra red-shifts accordingly. For the lowest dose array, the total exposure time is $\sim 45-60$ s. At d (iii, iv), the transmission spectra provides the narrowest and most efficient transmission filtering over visible wavelengths. These are designed to be $130 \mathrm{~nm}$ and $165 \mathrm{~nm}$ linewidths respectively. Interestingly, at smaller designed linewidths and larger duty cycles, along with the red shifting, multiple resonances are observed (d, v - vi) indicative of a hybridsation of multiple resonant electromagnetic modes $[1,31,32]$. Due to the $22 \mathrm{~nm}$ thick Ag layer, the optically vivid colours in transmission are a variant of extraordinary optical transmission (EOT) [33], termed extraordinary low transmission (ELT) $[1,31,32]$. The ELT effect stems from the excitation and hybridization of SPP mode, and LSPR of the optically thin metal grating, leading to a pronounced dip in the transmission spectrum. ITO has a plasma frequency $\sim 1 \mathrm{eV}$ (near infrared), and above this, in the visible, the response can be considered dielectric in nature [34]. Its refractive index is higher than that of quartz across the visible spectrum and hence, due to the influence of the local surrounding dielectric on the plasmonic excitation, ITO will act to red-shift the resonant plasmon mode. This simple assumption does not consider a number of other mechanisms involved, for example the surface roughness of ITO which would increase electron scattering at the interface. However, the influence of ITO and exact mechanisms involved with the optical properties of the system are beyond the scope of the work presented here.

As the dose increases, the gratings with larger linewidths, $L$, and duty cycles, $\Gamma$, 
(a)

Device

photos
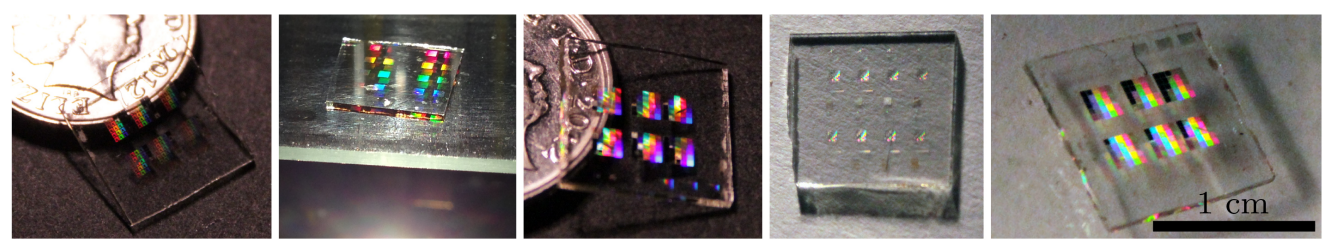

(b)

Isolated structures
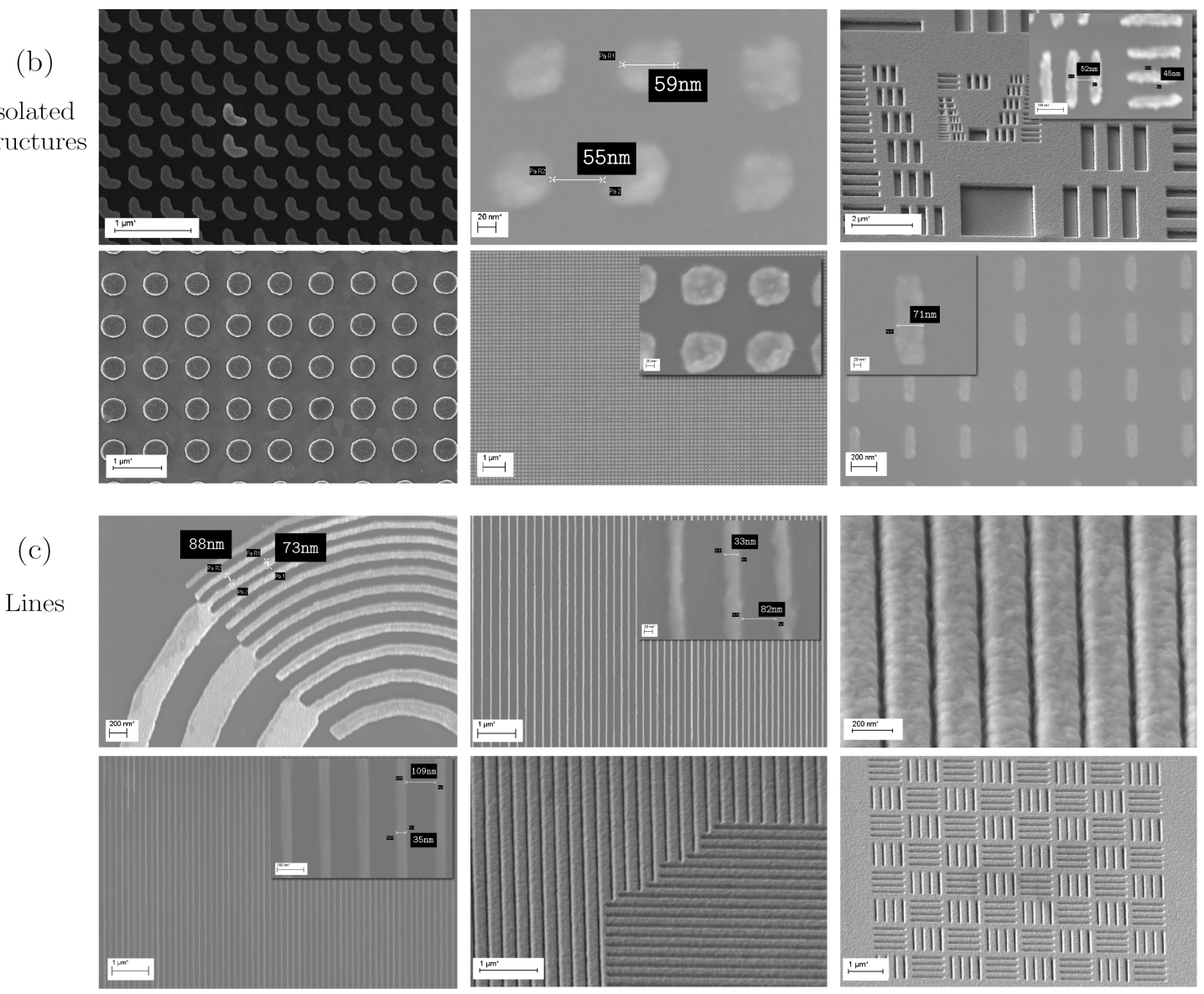

Figure 2. UV1116 (0.5) resolution capability examples. (a) Optical images of a range of ITO-glass samples used showing a range of patterns. The pieces of glass are $\sim 1 \mathrm{~cm}^{2}$. (b) SEM images of a range of isolated features, these are: an array of L-shaped optical antennas; nanostructure dot arrays of varying dimensions and spacings for spectral filters; anisotropic nanostructure rods for polarization dependent spectral filtering; and a USAF resolution test grid of decreasing feature sizes, before and after lift-off (inset). (c) 1D line arrays, with measurement estimations taken during characterization. The patterns include: 1D line gratings of varying grating widths and duty cycles; curved line gratings with decreasing grating widths (for resolution purposes); and arrays of sub-pixel orthogonal gratings (pre-lift-off) for spatial polarization dependent filtering. 
(a)

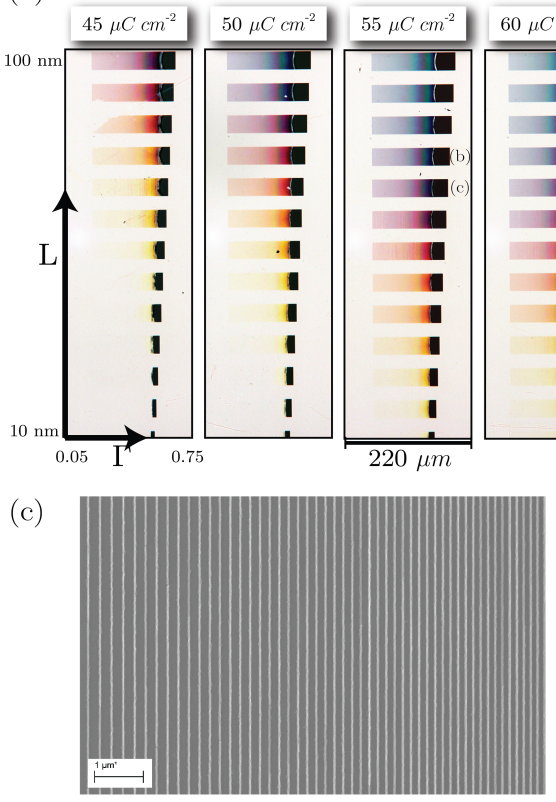

$60 \mu \mathrm{C} \mathrm{cm}^{-2}$
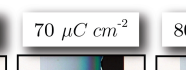

$80 \mu \mathrm{Ccm}^{-2}$
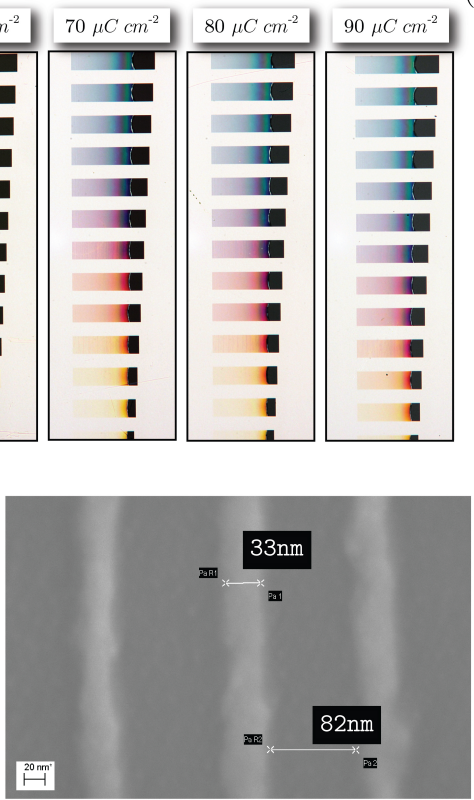

(b)
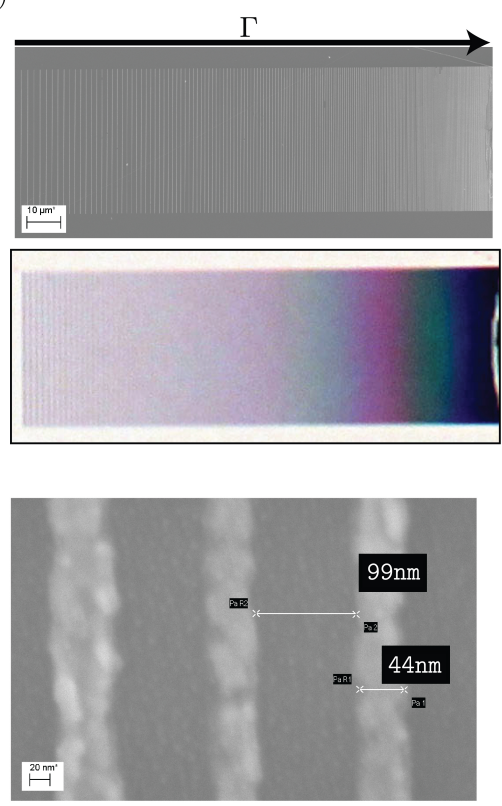

Figure 3. UV1116(0.5) resolution test: Logarithmically spaced 1D line arrays with varying linewidths, with 7 varying exposure doses, at 12s developing time (a) Optical microscope images (polarised parallel to grating vector) of the dose test. (b) SEM image of specific array shown in (a) with optical counterpart. (c) Shows an example of patterns under SEM
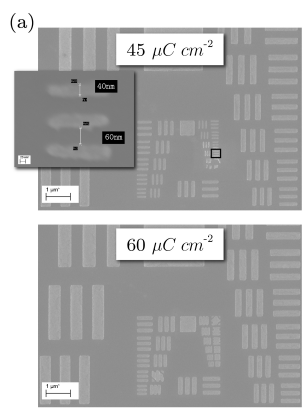
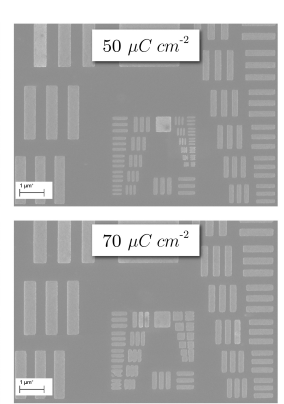
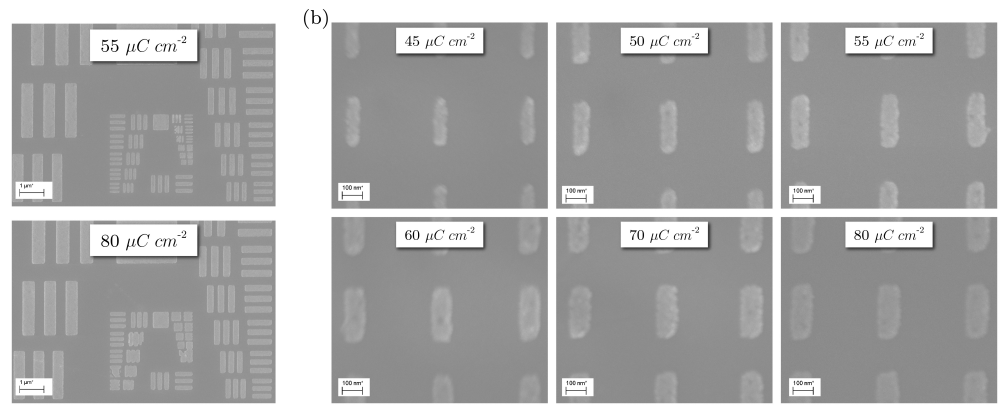

Figure 4. Isolated feature resolution testing through dose test comparison. (a) SEM images of USAF resolution test grids with rulings down to $25 \mathrm{~nm}$ linewidths, and (b) an array of nanorods with designed lateral dimensions $75 \times 200 \mathrm{~nm}$

are over-exposed and as a result, the entire grating lifts off; top left of the images in (c). For sparser lines (same linewidths), the larger doses results in a red shift in spectra i.e. larger linewidths. The designed linewidth limits were 75 and $220 \mathrm{~nm}$. For a dose of $45 \mu \mathrm{C} \mathrm{cm}{ }^{-2}$, the fabricated linewidths were measured under SEM at a variety of positions along the gratings at $\sim 60-80 \mathrm{~nm}$ and $\sim 215$ - $225 \mathrm{~nm}$ respectively - in relation to Figure 5 (b) 1 and 2. Therefore the resolution capability over areas $\sim 100 \mu \mathrm{m}^{2}$ appears consistent. The realised linewidth, in comparison to designed, has a geometric variation which reduces as the linewidth and duty cycle increases. In (e), we take the 


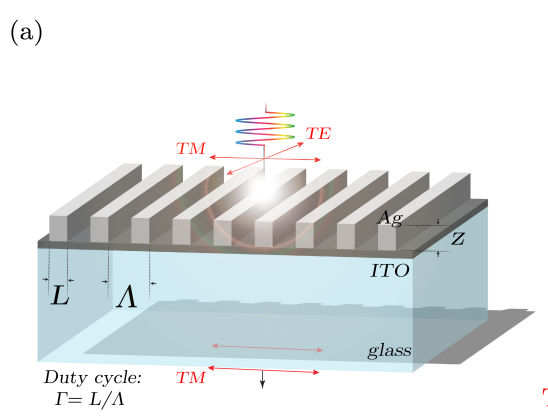

(b)
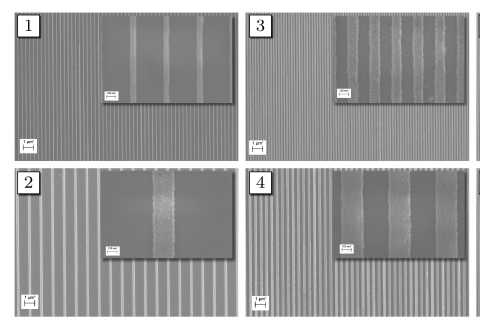

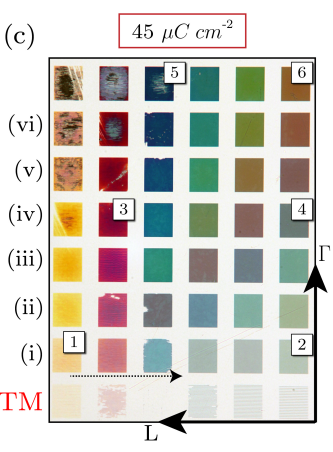

(d)

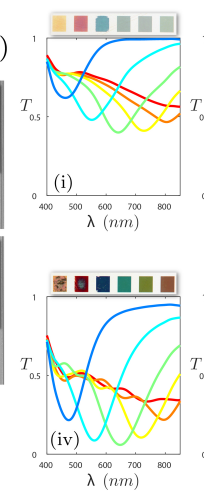

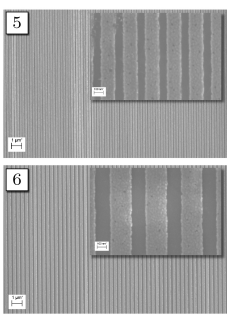
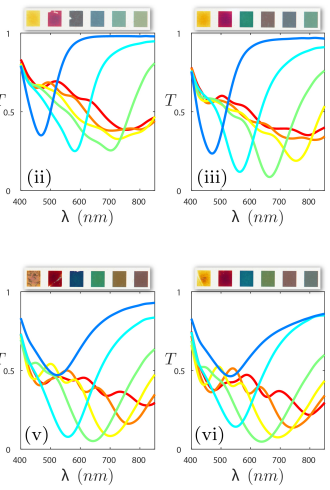

(e)

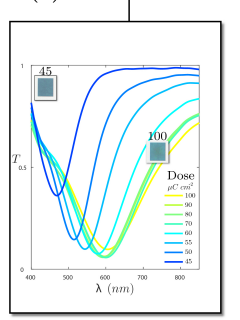

Figure 5. An array of $\mathrm{Ag}(22 \mathrm{~nm}) 1 \mathrm{D}$ gratings with varying linewidths and duty cycles. (a) Schematic of 1D grating with labeled iterated parameters. (b) SEM images of a selection of gratings: 1-6 correspond to gratings 1-6 in the optical images in, (c): which shows the optical images of the large array of gratings with varying parameters over a range of doses. (d) Shows the transmission spectra of a section of 6 rows from dose $45 \mu \mathrm{C} \mathrm{cm}^{-2}$. (e) Transmission spectra for the highlighted grating over a range of doses

spectra for the same grating as a function of dose. The resonance dip increases and red shits as dose increases, implying the linewidths are larger and hence effective duty cycle increases. Hence, area dose is highly critical, not only for realising minimum feature sizes but also for ensuring the fabricated geometries match that of designed.

\subsection{Polarising resolution test targets}

For many optical applications, resolution test targets are critical components. Here, we extend testing the resolution capability of the resist with orthogonal 1D gratings to demonstrate novel polarisation-dependent resolution test targets (chromatic and broadband operation) based on thin-film metallic gratings. Wire grid polarizers (WGPs) consist of arrays of parallel metallic wires with periodicity $<\lambda$. Generally, subwavelength gratings consist of either spectrum filters or polarising filters. The former subtractively filters specific wavelengths at certain polarisation states (normally due resonant SPP mode-coupling) the latter, operates beyond the cut-off of the device, is heavily sub-wavelength and merely acts to polarise the reflected/transmitted light $[4,30,31]$. The former is highlighted by the Al WGPs in Figure $6(\mathrm{c}, \mathrm{e})$ and the latter 


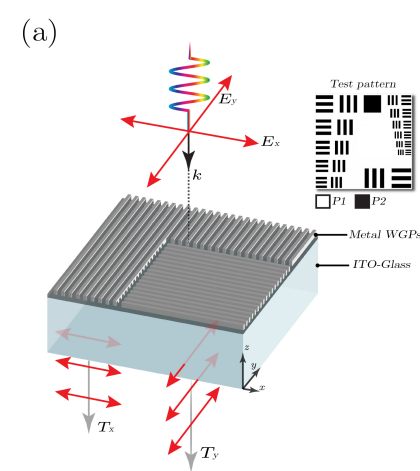

(c) $\quad \mathrm{Al}(\mathrm{z}=120 \mathrm{~nm})$

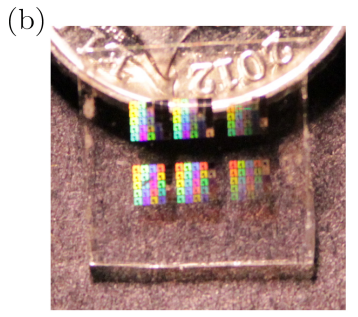

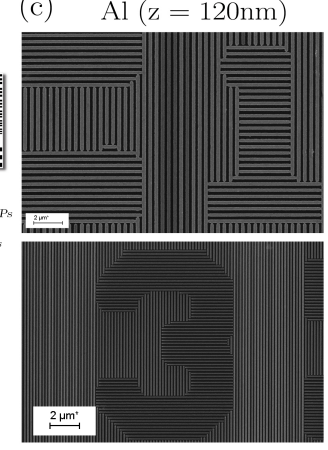

(d)

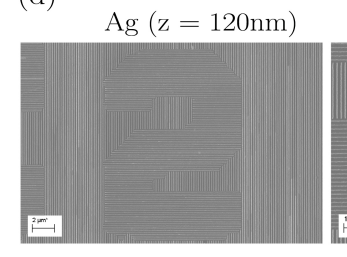

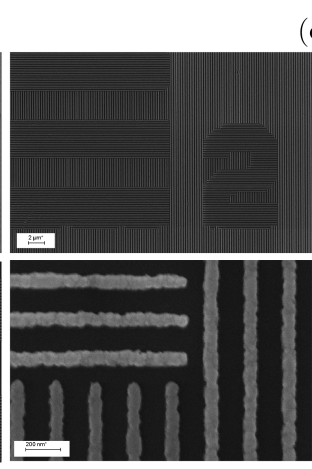

(f)

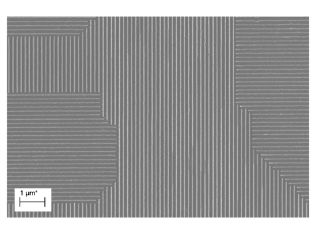

(e)
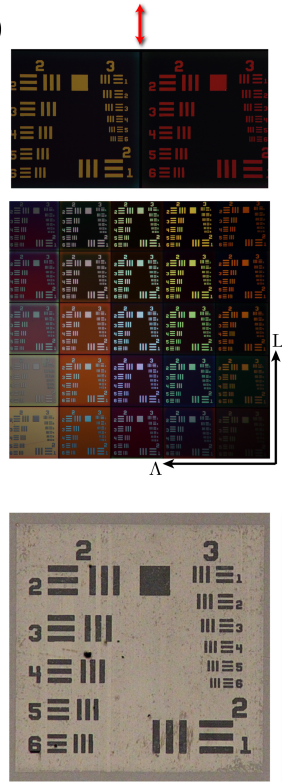

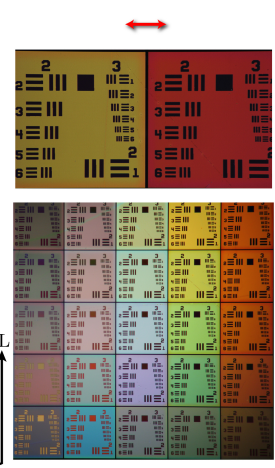

$0.75 \mathrm{~mm}$

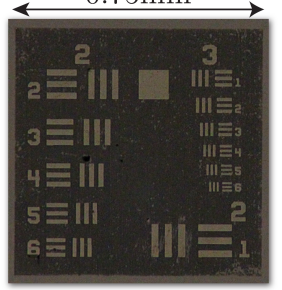

Figure 6. Polarisation sensitive resolution test targets. (a) Schematic of operating principles and (b) optical image of a typical sample with the 5x5 USAF grids against a coin for scale to illustrate the total area patterned. USAF resolution test targets as polarisation dependent spectral filters $(\mathrm{c}, \mathrm{e})$ or broadband polarisers $(\mathrm{d}, \mathrm{f})$

by the Ag WGPs in (d,f).

The devices here are polarization sensitive transmissive test grids based on the 1951 USAF resolution test chart. The test pattern is composed of orthogonal wires (rulings) at a range of decreasing linewidths and spacings. For the spectral polarisation filters, an array of gratings with varying linewidths and duty cycles are fabricated Figure 6 (c,e). As duty cycle decreases (line spacing increases), the colour filtering associated with the resonant plasmonic mode is more pronounced. Conversely, for a broadband WGP $(\mathrm{d}, \mathrm{f})$, the designed linewidth is decreased $(<100 \mathrm{~nm})$ and duty cycles are increased, resulting in broadband (white-black) states in orthogonal polarizing conditions. For the $5 \times 5$ array,

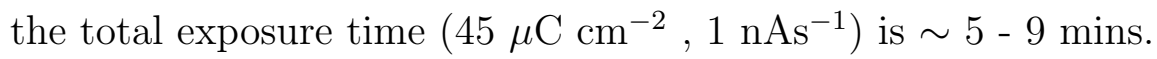

\subsection{Transmission polarisation dependent plasmonic nanostructures}

Transmission plasmonic filters are based on an ultra-thin nanostructure arrays and with spacings much smaller than the wavelength of light. They provide color filtering at much smaller length scales than that of conventional color (pigment-based) filters [1] . Each nanostructure acts as a dipole scatterer, whose polarizability is geometry dependent. For the total array, the response is given by a summation of scatterers, whereby the periodicity and unit cell also determine spectral and amplitude response characteristics $[1,6,35]$. For an anisotropic geometry, longitudinal and transverse LSPRs can be excited and selected with different polariser states. Using UV1116, we produced arrays of Ag $(22 \mathrm{~nm})$ anisotropic nanostructures for color filters with a range of geometries: width 
fixed at $75 \mathrm{~nm}$, length varying from $75-260 \mathrm{~nm}$, symmetric unit cells (characterized with duty cycles from $0.2-0.6)$, over a range of exposure doses $\left(45-100 \mu \mathrm{C} \mathrm{cm}^{-2}\right)$ and development times $(12-60 \mathrm{~s})$. In Figure (6), 3 exposure doses and the $12 \mathrm{~s}$ development sample is chosen once more, due to superiority in optical inspection.

Figure 7 shows the results for both transverse (c-d) and longitudinal (e-d) polarisation states in transmission. For the lowest dose, the designed width is $75 \mathrm{~nm}$, with experimentally realized sizes varying from $70-80 \mathrm{~nm}$. The results in $(\mathrm{g})$ show how this varies with exposure dose, whereby the increase in dose leads to larger isolated features $\left(100 \mu \mathrm{C} \mathrm{cm}^{-2}\right.$, leads to an increase of $\left.\sim 40-50 \mathrm{~nm}\right)$, which, results in smaller spacing and increase in transmission dip. In (c), for a fixed duty cycle (unit cell), as length increases, the transverse mode should remain fixed spectrally, however the amplitude will vary with spacing. In the low dose scenario, this is seen (d, i-iii, top), however with a high dose (d,i -iii, bottom), the spectral positions vary, indicating an increase in lateral feature size.

For longitudinal polarisation (e-f), only the shorter rod lengths (iv - vi) can be acquired on the UV-vis spectrometer. This is because at a thickness of $22 \mathrm{~nm}$, the LSPR is red shifted (in comparison to thicker nanostructures) such that the response is beyond the spectrometer cut-off in the NIR. Here, the spectra is taken with a fixed design length and varying duty cycles. As before, with a large dose (f, iv - vi, bottom) the spectral position changes indicating a widening of the features and the decrease in spacing (increase in duty cycle) leads to a more pronounced transmission dip. In (g), we corroborate the SEM size information with optical spectra. Whereby, two color arrays are observed and characterised over a range of doses. A larger dose leads to larger features, leads to an effective reduction in spacing, and hence the spectra shifts its peak position and increases its transmission dip. For the transmission plasmonic filter array, the total exposure time $\left(45 \mu \mathrm{C} \mathrm{cm}{ }^{-2}, 1 \mathrm{nAs}^{-1}\right)$ is $\sim 10-30 \mathrm{~s}$.

\subsection{Plasmonic holograms}

Binary amplitude computer generated holograms (CGHs) encoded with plasmonic nanostructures, shown in Figure 8, are designed using methodologies described in previous work $[2,36]$ and fabricated on ITO-glass using the conditions described above.Figure 8 (c) shows the target diffraction pattern (replay-field) associated with each hologram. Because the nanostructured hologram is composed of sub-wavelength metallic scatterers, whereby each element emanates radiation in all directions, (cbottom) shows a mapping of the replay-field to the surface of a sphere, indicating the expected intensity distribution if captured over an imaginary sphere. In reality, the radiation is shown by a diffuse screen which effectively acts as a plane intersecting this sphere of radiation. This effect can be seen due to the distortion of the replay-field in (d) and target pattern in (b). Upon Illumination with green (523 nm) and red (650 nm) laser diodes (spot size $\sim 0.5 \mathrm{~cm}^{2}$ ) the far-field replay-field associated with the holograms can be observed in both transmission and reflection - as seen in (d). The extremely wide- 


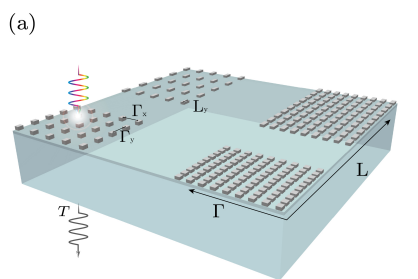

(b)

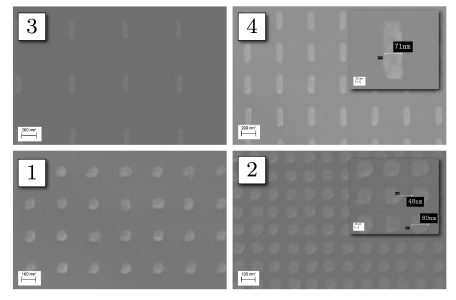

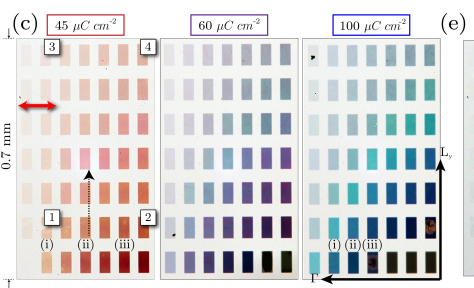

(d)

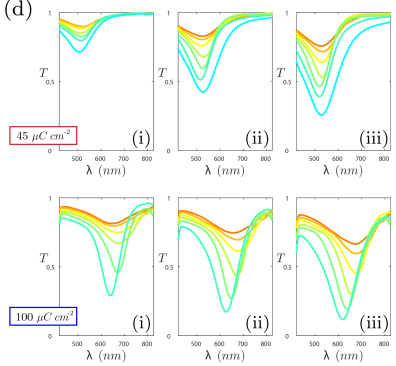

(f)

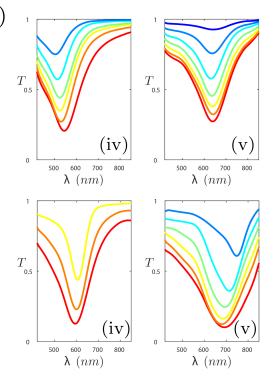

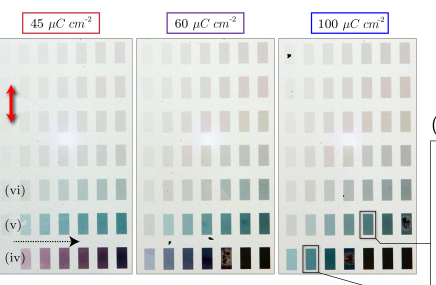

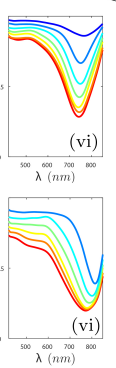

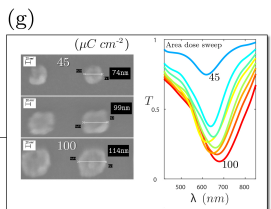

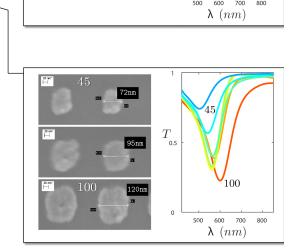

Figure 7. Polarisation dependent anisotropic nanostructured array experimental results. (a) Schematic of the large array and how the isolated features vary with position. (b) SEM images of 4 parts of the total array; in reference to the optical images in (c). (c-d) show the optical images and spectra of a range of nanostructure arrays under transverse polarisation state; cyan-to-orange is bottom-to-top in optical image. Longitudinal polarisation is shown in (e-f), blue-to-red is left-to-right in optical image. (g) Shows both SEM and optical spectra of the same part of the total array but with results from the dose test, showing how the geometry varies with dose

angle replay-fields of these devices are associated with the nanostructured size of the scattering elements and spacing, whereby the maximum diffraction angle from an array of scatterers can be approximated by Bragg's law, such that, as feature size decreases, the maximum diffracted angle increases. Because the overall exposure area for the array of scatterers is small, the write-time is small, e.g. the total exposure time $\left(50 \mu \mathrm{C} \mathrm{cm}^{-2}\right.$, $1 \mathrm{nAs}^{-1}$ ) is $\sim 4 \mathrm{~s}$.

\section{Conclusions}

Nanostructured transmissive optical elements offer potential benefits in a wide range of disciplines and with the growing trends toward plasmonc-enhanced devices, whereby nanoscale geometries are essential to achieve unique spectral signatures, high-resolution fabrication over large-areas on insulators is required. Conventional high-energy EBL fabrication on insulating substrates for sub-wavelength dense feature, large-area patterns with high throughput can be challenging. In this work we have demonstrated the use of UV1116 [Dow Chemical Company] photoresist on ITO-glass for high resolution EBL processing. UV1116 presents an attractive alternative to conventional ebeam resists for patterning on insulating substrates due to its low exposure sensitivity (critical dose), maximum resolution and throughput, and thus one is able to utilize a reasonable exposure current, without resulting in charge build-up issues found in conventional EBL resists. The minimum realised linewidth in this work was $\sim 33 \mathrm{~nm}$ with $80 \mathrm{~nm}$ spacing. 

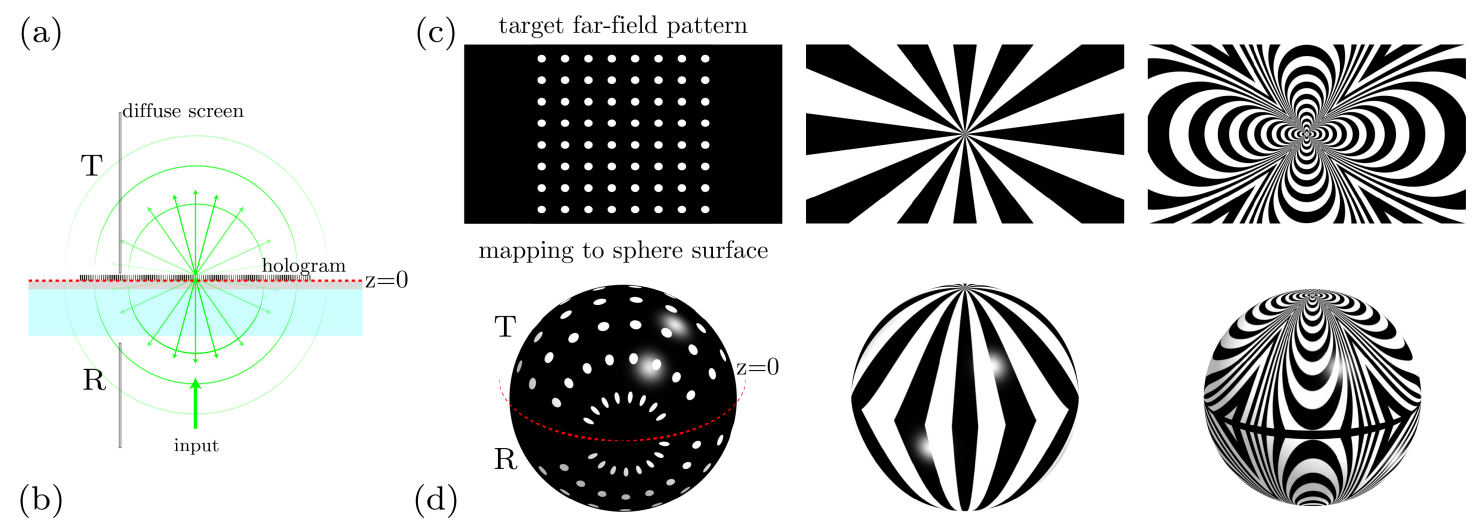

(d)

mapping to sphere surface
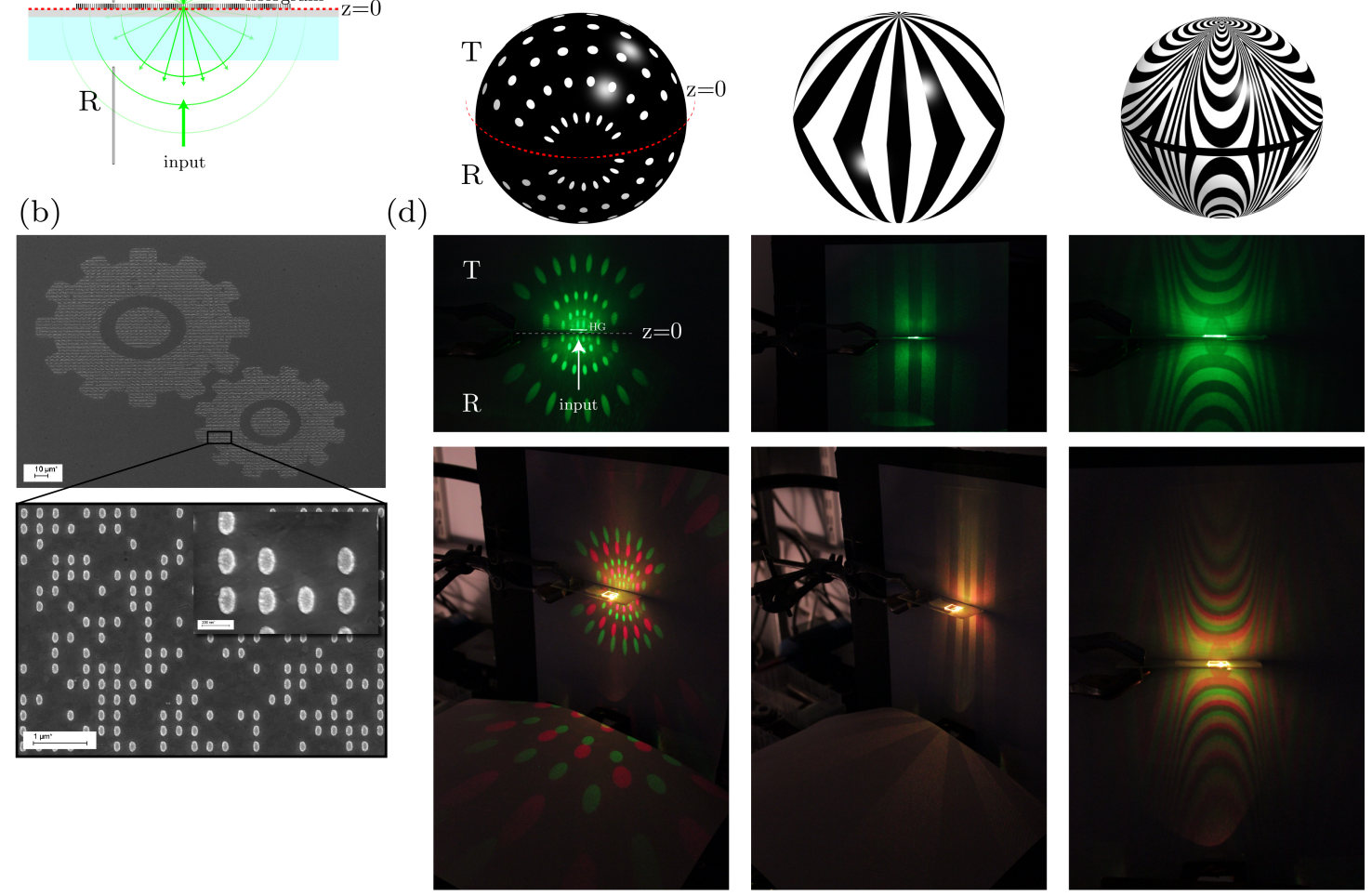

Figure 8. Plasmonic holograms patterned on ITO-glass. (a) Schematic of illumination set-up showing reflection and transmission of hologram (HG) sample. (b) Example of a hologram whereby the hologram is encoded with Ag plasmonic nanostructures cropped into two gear arrangements. The ITO grains can be clearly seen underneath the higher magnification image. Three hologram examples: (top) the target far-field image with mapping to spherical surface (c), (middle) reflection and transmission with green (532 $\mathrm{nm}$ ) laser diode and (bottom) with red $(650 \mathrm{~nm})$ and green laser diode simultaneously, showing the far-field pattern scattering off diffuse white paper (d)

The lowest dose ( $45 \mu \mathrm{C} \mathrm{cm}^{-2}$, beam current $1 \mathrm{nAs}^{-1}, 12 \mathrm{~s}$ developing time) yielded the highest resolution but reproducibility was limited. However smaller linewidths, with large spacings were hard to achieve with the lower dose. For isolated structures, $\sim 45$ $\mathrm{nm}$ structural width and $50 \mathrm{~nm}$ separation were achieved. An area dose of $50-60$ $\mu \mathrm{C} \mathrm{cm}^{-2}$ offered more reproducible results over larger areas but meant a reduction in resolution (lateral feature expansion). The sparsely spaced lines were more reproducible at higher beam energies, but broadened. Approaching $100 \mu \mathrm{C} \mathrm{cm}^{-2}$, isolated features, separated by a minimum $\sim 100 \mathrm{~nm}$, were achievable, however the denser features were not.

To demonstrate resist suitability for transmissive optical devices, we fabricated a 
range of optical devices composed of sub-wavelength optical nanostructures, patterned on ITO-glass. These devices were based on silver nanostructures (22nm thick) whereby surface plasmon polaritons are excited through incident light, with distinct transmission characteristics dependent on geometrical factors and material properties. Through 1D and 2D silver arrays, we observed strong resonant behaviour through dips in the transmission spectrum. The throughput was relatively high (exposure time low) and the work highlights the potential of UV1116 photoresist for larger scale optical applications using EBL as the fabrication technique.

Further optimization of area dose, development time and more significantly beam current, should lead to greater throughput and improved resolution. The beam current condition was not optimized in this study. Good quality, low surface roughness ITO coverage is essential, along with proper earthing of the surface of the sample to an electrical ground plate. In addition, the resist film thickness is $\sim 350 \mathrm{~nm}$, (due to the

viscosity 0.5 type resist) and hence the resolution is improved further by choosing a UV1116 series with a higher solvent concentration and thus reducing nominal film thickness. A low current both reduces the effect of charge-build up and beam spot size, yet there is a trade-off over total write time. As a comparison, the area dose used for UV1116 (0.5) is approximately an order-of-magnitude less than the critical exposure dose for PMMA / HSQ at $80 \mathrm{kV}$ and throughput can be much greater.Nevertheless, clearly PMMA and HSQ with intrinsic resolutions of $<10 \mathrm{~nm}$ offers superior resolution capability. However, for larger scale optical applications where this level of resolution is not required, UV1116 offers an alternative attractive solution.

\section{Acknowledgments}

EPSRC Integrated Photonics and Electronic Systems (Grant number: EP/L015455/1) Centre for Doctoral Training. Commonwealth, European and International trust. The authors would like to thank James Dolan, Ammar Khan and Alexander Macfaden for their insights, discussion and help during the work.

\section{References}

[1] Gu Y, Zhang L, Yang J K W, Yeo S P and Qiu C W 2015 Nanoscale 7 6409-19

[2] Williams C, Montelongo Y, Tenorio-Pearl J O, Cabrero-Vilatela A, Hofmann S, Milne W I and Wilkinson T D 2015 physica status solidi (RRL) - Rapid Research Letters 5 1-5

[3] Genevet P and Capasso F 2015 Reports on Progress in Physics 78024401

[4] Xu T, Wu Y K, Luo X and Guo L J 2010 Nature communications 159

[5] Yu N and Capasso F 2014 Nature Materials 13 139-150

[6] Maier S 2007 Plasmonics: Fundamentals and Applications (Boston, MA: Springer) ISBN 978-0387-33150-8

[7] Takigawa T, Kawabuchi K, Yoshimi M and Kato Y 1983 Microelectronic Engineering 1 121-142

[8] Shawn C, Makiuchi Y and Che C 2010 High-energy Electron Beam Lithography for Nanoscale Fabrication Lithography February ed Wang M (InTech) ISBN 978-953-307-064-3

[9] Grigorescu A E and Hagen C W 2009 Nanotechnology 2031 
[10] Muhammad M, Buswell S C, Dew S K and Stepanova M 2011 Journal of Vacuum Science 83 Technology B: Microelectronics and Nanometer Structures 29 06F304

[11] Mohamed K, Alkaisi M M and Blaikie R J 2009 Microelectronic Engineering 86 535-538

[12] Alexander Liddle J, Gallatin G M and Ocola L E 2011 MRS Proceedings 739 H1.5

[13] Chen Y 2015 Microelectronic Engineering 135 57-72

[14] Anderson E H, Olynick D L, Chao W, Harteneck B and Veklerov E 2001 Journal of Vacuum Science \&5 Technology B: Microelectronics and Nanometer Structures 192504

[15] Dong Z, Bosman M, Zhu D, Goh X M and Yang J K W 2014 Nanotechnology 25135303

[16] Thyagarajan K, Santschi C, Langlet P and Martin O J F 2016 Advanced Optical Materials 4 $871-876$

[17] Salerno M and Cingolani R 2007 Journal of Micromechanics and Microengineering 17 2414-2419

[18] Samantaray C B and Hastings J T 2008 Journal of Vacuum Science \& Technology B: Microelectronics and Nanometer Structures 26 2300-2305

[19] Dylewicz R, Lis S, De La Rue R and Rahman F 2010 Electronics Letters 461025

[20] Mohammad M A, Muhammad M and Dew S K 2012 Nanofabrication (Vienna: Springer) ISBN 978-3-7091-0423-1

[21] Zhang J, Fouad M, Yavuz M and Cui B 2011 Microelectronic Engineering 88 2196-2199

[22] Pépin A, Studer V, Decanini D and Chen Y 2004 Microelectronic Engineering 73-74 233-237

[23] Bilenberg B, Jacobsen S, Schmidt M S, Skjolding L H D, Shi P, Bøggild P, Tegenfeldt J O and Kristensen A 2006 Microelectronic Engineering 83 1609-1612

[24] Lu, Bing-Rui; Chen, Yifang; Huq, Ejaz; Qu, Xin-Ping; Liu R 2010 Journal of Nanoscience and Nanotechnology 10 7130-7133(4)

[25] Joo J, Chow B Y and Jacobson J M 2006 Nano Letters 6 2021-2025

[26] Wiederrecht G P 2009 Handbook of Nanofabrication (Academic Press)

[27] Pala N and Karabiyik M 2010 Electron Beam Lithography Encyclopedia of Nanotechnology 2007 (Springer) pp 718-740

[28] Tseng A A, Chen K and Chen C D 2003 IEEE Transactions on Electornics Packaging Manufacturing 26 141-149

[29] Drouin D, Couture A R, Joly D, Tastet X, Aimez V and Gauvin R 2007 Scanning 29 92-101

[30] Zhou J and Guo L J 2014 Scientific reports 43614

[31] Zeng B, Gao Y and Bartoli F J 2013 Scientific Reports 3 1-9

[32] Reibold D, Shao F, Erdmann A and Peschel U 2009 Optics Express 17544

[33] Ghaemi H F, Thio T, Ebbesen T W, Lezec H J, Ghaemi H F, Thio T and Wolff P A 1998 Nature 391 667-669 (Preprint 0008204)

[34] Rhodes C, Franzen S, Maria J P, Losego M, Leonard D N, Laughlin B, Duscher G and Weibel S 2006 Journal Of Applied Physics 10054905

[35] Park W 2014 Nano Convergence 12

[36] Montelongo Y, Tenorio-Pearl J O, Williams C, Zhang S, Milne W I and Wilkinson T D 2014 Proceedings of the National Academy of Sciences 111 12679-12683 